\title{
Fatores associados à infecção de sítio cirúrgico em pacientes idosos submetidos à cirurgia cardíaca com esternotomia
}

\author{
Elia Machado Oliveira ${ }^{1}$ Josue Bruginski de Paula²
}

\begin{abstract}
RESUMO
Introdução - Infecção de Sítio Cirúrgico (ISC) em cirurgia cardíaca constitui uma grave complicação no pós-operatório, desafiando as equipes de saúde, apesar dos avanços da medicina e das técnicas cirúrgicas. Objetivo - Avaliar a associação entre preditores em pacientes submetidos a cirurgias cardíacas em um serviço de cirurgia cardíaca na cidade de Curitiba. Metodologia - Realizado nos anos de 2007/2008 com 42 pacientes, divididos em 02 grupos, pareados por idade e gênero. Submetidos a 03 consultas de enfermagem para coleta de informações, realizadas no pré-operatório, pós-operatório imediato e na quarta semana de cirurgia. Resultados - Uso de concentrado de hemácias apresentou significância estatística, favorecendo ISC ( 0,005$)$. Na análise do risco relativo detectou-se que pacientes tabagistas tiveram 2.33 vezes mais chances de infecção. Diabetes, internamento prolongado ou obesos também tiveram mais ISC. Conclusão - Diabetes, internamento prolongado pré-operatório, tabagismo, obesidade e uso de concentrado de hemácias, na população estudada, favoreceram o risco para ISC em cirurgias cardíacas.
\end{abstract}

Descritores: Cirurgia Cardíaca, Infecção.

\section{Factors associated with surgical site infection in elderly patients undergoing cardiac surgery with sternotomy}

\begin{abstract}
Introduction - Surgical Site Infection SSI in cardiac surgery is a serious complication in the postoperative period; it challenges health teams, despite advances in medicine and surgical techniques. Objective - To evaluate the association between predictors in patients undergoing cardiac surgery in a cardiac surgery service in Curitiba. Methodology - Conducted in the years 2007/2008 with 42 patients divided into 02 groups, matched by age and gender. They were subjected to 03 nursing visits for information gathering which were performed preoperatively, immediately postoperatively and in the fourth week after surgery. Results - The use of Concentrate Erythrocytes showed statistical significance, favoring SSI p 0.005. In the analysis of the relative risk was found that smokers had 2:33 times more chances of infection. Diabetes, prolonged hospitalization or obese had also more SSI. Conclusion - Diabetes, prolonged preoperative hospitalization, smoking, obesity and use of use of red blood cells concentrate in the population studied favored the risk for SSI in cardiac surgery.
\end{abstract}

Descriptors: Cardiac Surgery, Infection.

\footnotetext{
${ }^{1}$ Mestre em cirurgia pela Pontifícia Universidade Católica do Paraná (PUC/PR), Curitiba, PR, Brasil.

${ }^{2}$ Doutorado em egenharia elétrica pela Universidade Estadual de Campinas (UNICAMP), Campinhas, SP, Brasil.
} 


\section{Introdução}

A infecção em pós-operatório, de maneira geral, é um sério problema, pois pode aumentar o tempo de internação, a letalidade, mortalidade e os custos hospitalares. Em cirurgia cardíaca, a infecção da ferida cirúrgica pode aumentar significativamente os custos, contribuir para a morbidade e até levar a morte ${ }^{1}$.

Considera-se a Infecção de Sítio Cirúrgico (ISC) a segunda em termos de incidência, sendo superada apenas pela infecção do trato urinário. Diversos fatores têm sido relacionados à incidência de ISC: referentes ao microrganismo, relacionados ao paciente, doenças pré-existentes, longo período de hospitalização pré-operatória, estado nutricional e fatores relacionados ao procedimento cirúrgico ${ }^{2}$.

Diante deste contexto, se deve considerar que a idade média de candidatos à cirurgia coronariana aumentou de 50 anos, em 1967, para 66 anos nos dias atuais. Estima-se que aproximadamente $30 \%$ dos pacientes têm mais de 70 anos de idade. $O$ risco de adquirir infecção de sítio operatório aumenta com a idade. Pacientes abaixo de 50 anos, entre 50 e 70 anos e acima de 70 anos apresentam incidência de 0,9\%, 2,7\% e 3,1\% respectivamente 2 .

Embora se discuta a discrepância entre as idades cronológica e fisiológica, a habilidade de idosos em responder à cirurgia cardíaca é menor, devido às comorbidades associadas, a limitada reserva funcional de órgãos vitais e a diminuição da capacidade de defesa e adaptação. Com o desenvolvimento de melhores técnicas de proteção miocárdica e pulmonar, além de avanços na avaliação pré-operatória, os benefícios da cirurgia cardíaca nos idosos vêm se estendendo para a oitava e nona décadas com maior frequência. Entretanto, os instrumentos de avaliação de risco cirúrgico ainda consideram a idade avançada como fator associado à maior morbimortalidade hospitalar.

O momento da indicação da cirurgia é um dos instantes importantíssimos para o pós-operatório do paciente. São neste momento que é identificado e relatado ao paciente todos os riscos do procedimento ao qual será submetido, inclusive o risco de infecção. Desta forma, há necessidade crítica para identificar os pacientes a serem submetidos a uma cirurgia cardíaca que correm o risco de infecções graves, permitindo o desenvolvimento e a adoção de intervenções eficazes para prevenção. Além do benefício direto ao paciente com uma rápida recuperação na ausência do quadro infeccioso, estas medidas preventivas também teriam impacto na redução dos custos que hoje são destinados ao tratamento de pacientes com infecção pós-operatória, pois parte destes não viria a apresentar esta condição após a adoção de medidas suplementares por toda a equipe de saúde envolvida³.

Estudos anteriores já identificaram fatores de risco para infecções no pós-operatório em cirurgia cardíaca, sendo que várias destas características, infelizmente, não podem ser determinadas antes da cirurgia ${ }^{3-4}$.

Diante desta problemática, este estudo teve como objetivo investigar a associação entre as variáveis preditoras de infecção e a manifestação de infecção de sítio cirúrgico esternal, em pacientes submetidos a cirurgias cardíacas com esternotomia longitudinal em um serviço de cirurgia cardíaca na cidade de Curitiba, Paraná.

\section{Metodologia}

Tratou-se de um estudo de coorte prospectivo, analítico, a partir de variáveis obtidas na consulta de Enfermagem no pré e pós-operatório de pacientes submetidos à cirurgia cardíaca, em um serviço especializado de cirurgia cardíaca. As cirurgias foram realizadas por uma mesma equipe médica, no período de outubro de 2007 a dezembro de 2008, em hospital privado de médio porte, com procedimentos custeados por planos de saúde.

O estudo foi aprovado pelo Comitê de Ética e Pesquisa com Seres Humanos do Hospital Erasto Gaertner, sob o número 1533/2007 que presta serviço a Instituição onde foi desenvolvido o trabalho. Os indivíduos foram inseridos no estudo após assinatura do Termo de Consentimento Livre Esclarecido, conforme Resolução 196/1996.

No primeiro momento foram analisados 130 pacientes para posterior pareamento por idade e gênero. Os dados relacionados ao perfil clínico foram analisados quanto à presença de patologias ou comorbidades associadas, como: hipertensão; diabetes mellitus; tabagismo ou ex-tabagismo; presença de obesidade; baixa fração de ejeção do ventrículo esquerdo e insuficiência renal.

Como variáveis relativas à cirurgia foram consideradas: o número de dias de internamento pré-operatório; tipo de cirurgia (cirurgia valvar ou de revascularização do miocárdio); uso de circulação extracorpórea; tempo de cirurgia; infusão de hemocomponentes durante a cirurgia ou nas primeiras 48 horas após 0 ato cirúrgico. 
Como critérios de inclusão consideraram-se: ter sido submetido à cirurgia cardíaca de revascularização miocárdica ou troca valvar na instituição onde o estudo estava sendo desenvolvido, possuir mais que 60 anos de idade, (terceira idade de acordo com estatuto do idoso) e residir no estado do Paraná.

Como critérios de exclusão adotaram-se: apresentar sequelas incapacitantes de acidente vascular cerebral, residir fora do estado do Paraná, indisponibilidade para realizar retorno ambulatorial na quarta semana, estar em vigência de infecção de qualquer natureza no pré-operatório, ser submetido à cirurgia de emergência, possuir Doença Pulmonar Obstrutiva Crônica (DPOC) estadiamento IV (doença muito grave), ter se submetido à cirurgia valvar e de revascularização do miocárdio no mesmo procedimento.

As consultas de Enfermagem foram realizadas no dia que antecedeu a cirurgia, no segundo dia de pós-operatório e no retorno ambulatorial na quarta semana de pós-operatório. O formulário impresso, utilizado e preenchido em cada etapa, seguia juntamente com o prontuário do paciente até o momento da última consulta.

$\mathrm{Na}$ consulta inicial foram coletados dados que antecederam o ato cirúrgico, como: data de internamento, gênero, idade, peso/altura, Índice de Massa Corporal (IMC), doenças pré-existentes (diabetes, hipertensão, tabagismo ou ex-tabagismo, insuficiência cardíaca por meio da Fração de Ejeção do Ventrículo Esquerdo (FEVE), DPOC, doença renal crônica, aferição da Pressão Arterial (PA) e histórico de alergias.

Na segunda consulta foram coletadas informações sobre a cirurgia e os dois primeiros dias de pós-operatório. Os dados coletados neste momento foram: cirurgia realizada (valvar ou revascularização); quantidade de dias de internamento que antecederam o ato cirúrgico; hora de inicio e do final da cirurgia (tempo de cirurgia); uso de Circulação Extracorpórea (CEC); uso de hemocomponentes (tipo e quantidade utilizada).

A terceira consulta foi realizada na quarta semana de pós-operatório, consistindo em novo exame físico direcionado, além de serem observados sinais flogísticos na cicatriz cirúrgica esternal. Na presença de algum sinal flogístico, ou na presença de secreção, realizou-se coleta de material de exsudação para cultura, assim como o encaminhamento ao cirurgião para avaliação e conduta.

A infecção foi definida com base em critérios clínicos: surgimento de sinais flogísticos, drenagem de exsudação, endurecimento das incisões, deiscência de sutura, presença de serosidade na ferida operatória, critérios laboratoriais (leucocitose) e cultura positiva ${ }^{5}$. Os pacientes que tiveram alguma infecção de sítio cirúrgico, de qualquer gravidade, foram denominados de Grupo Infecção (GI). Os pacientes em que não houve infecção formaram o Grupo Não Infecção (GNI).

A partir da formação dos grupos (GI) e (GNI), optou-se pelo pareamento dos grupos por gênero e idade, admitindo-se uma variação de dois anos de idade para mais ou para menos, e tendo como base o grupo de menor tamanho.

Os dados foram obtidos pelo mesmo examinador, utilizando formulário de anamnese impresso, durante o período em que os pacientes permaneceram internados e no primeiro mês após a alta hospitalar.

Os resultados obtidos no estudo foram expressos por frequências e percentuais ou por médias, valores mínimos, valores máximos, desvios padrão e valor de $p$. Para a avaliação da associação entre variáveis nominais dicotômicas e a presença ou não de infecção de sítio cirúrgico, utilizou-se o Teste estatístico Exato de Fisher. Para comparação entre pacientes com infecção e pacientes sem infecção em relação a variáveis quantitativas utilizou-se o Teste $t$ de Student para amostras independentes. A condição de normalidade foi avaliada pelo Teste de Shapiro-Wilks. Para avaliação conjunta das variáveis foi ajustado um modelo de Regressão Logística (stepwise backward). Valores de $p<0,05$ indicaram significância estatística. Os dados foram organizados em planilha Exce ${ }^{\circledR}$ e analisados com 0 programa computacional Statistica v.8.0.

Para cada uma das variáveis nominais dicotômicas, testou-se a hipótese nula de que a probabilidade de ter infecção de sítio cirúrgico é igual para as duas classificações da variável, versus a hipótese alternativa de probabilidades diferentes, com teste de ODDS ratio para cada uma das hipóteses, por meio do software Epilnfo 6.04.

\section{Resultados}

Centro e trinta pacientes foram analisados, sendo que 21 foram diagnosticados com infecção e 109 não apresentaram infecção. Os pacientes com ISC compuseram o grupo $\mathrm{GI}(\mathrm{n}=21)$ e foram pareados por gênero e idade com 21 pacientes sem infecção, que formaram o grupo $\mathrm{GNI}(\mathrm{n}=21)$, totalizando 42 pacientes na amostra estudada. Para cada paciente foram registradas variáveis clínicas e cirúrgicas de interesse. 
Após o pareamento, a média de idade ficou idêntica nos dois grupos, ficando as duas populações homogêneas, valores mínimos iguais e valores máximos com dois anos de diferença a mais no Gl. Os desvios padrão foram semelhantes. Neste estudo os homens compuseram $66,67 \%$ de cada grupo, ficando as mulheres com $33,33 \%$. Pelo pareamento, a proporção entre homens e mulheres é idêntica nos dois grupos, mas houve predomínio do gênero masculino no $\mathrm{Gl}$.

O IMC médio foi de $27,43 \mathrm{Kg} / \mathrm{m} 2$ no $\mathrm{Gl}$ e de $26,46 \mathrm{Kg} / \mathrm{m} 2$ no GNI. Os valores máximos foram de $41,09 \mathrm{Kg} / \mathrm{m} 2 \mathrm{no} \mathrm{Gl}$ e de $32,59 \mathrm{Kg} / \mathrm{m} 2$ no GNI, ficando ambos os grupos abaixo dos valores considerados de obesidade mórbida. Na comparação entre os grupos GI e GNI o valor de p não apresentou significância estatística. A Tabela 1 apresenta estas variáveis.

Tabela 1 - Características pré-operatórias

\begin{tabular}{|c|c|c|c|c|c|c|}
\hline Preditor & Grupo & Média & DP & Mínimo & Máximo & Valor $p$ \\
\hline \multirow{2}{*}{ Idade } & $\mathrm{GI}$ & 70,33 & 7,89 & 61 & 92 & \\
\cline { 2 - 7 } & $\mathrm{GNI}$ & 70,23 & 7,62 & 61 & 90 & \\
\hline \multirow{2}{*}{ IMC } & $\mathrm{GI}$ & 27,43 & 4,34 & 21,48 & 41,09 & 0,456 \\
\cline { 2 - 7 } & $\mathrm{GNI}$ & 26,46 & 3,24 & 21,00 & 32,59 & \\
\hline \multirow{2}{*}{ FEVE } & $\mathrm{GI}$ & 61,38 & 9,42 & 40 & 73 & 0,499 \\
\cline { 2 - 7 } & $\mathrm{GNI}$ & 63,09 & 8,18 & 47 & 81 & \\
\hline
\end{tabular}

Desvio Padrão (DP); Grupo Infecção (GI); Grupo não Infecção (GNI); Indice de Massa Corpórea (IMC); Fração de Ejeção do Ventrículo Esquerdo (FEVE).

A fração de ejeção do ventrículo não apresentou diferença estatística significativa. As médias para os grupos foram de $61,38 \%$ no $\mathrm{Gl}$ e de $63,09 \%$ no GNI, com desvio-padrão de 9,42 e 8,18 respectivamente, ficando fora do limite de baixa ejeção (<50\%). O valor mínimo foi de $40 \%$ no Gl e de $47 \%$ no GNI. Os valores máximos foram de $81 \%$ no GNI e de $73 \%$ no Gl. O valor de p não demonstrou haver significância estatística entre as médias.

Os valores de pressão arterial não demonstraram ser um fator predisponente a infecção nestes pacientes estudados. Ambos os grupos apresentaram PAM máxima acima de $115 \mathrm{mmHg}$, havendo, neste parâmetro desvio padrão elevado para ambos os grupos. A Tabela 2 apresenta as variáveis relativas aos valores de pressão arterial dos grupos.

Tabela 2 - Valores da Pressão Arterial

\begin{tabular}{|c|c|c|c|c|c|c|}
\hline & \multicolumn{2}{|c|}{ PAS } & \multicolumn{2}{|c|}{ PAD } & \multicolumn{2}{|c|}{ PAM } \\
\hline & $\mathrm{GI}$ & GNI & $\mathrm{GI}$ & GNI & $\mathrm{Gl}$ & GNI \\
\hline Média & 135,71 & 136,33 & 85,23 & 80,47 & 101,71 & 98,66 \\
\hline Desvio Padrão & 19,95 & 13,59 & 11,56 & 9,34 & 12,37 & 9,31 \\
\hline Mínimo & 100 & 110 & 60 & 60 & 73 & 85 \\
\hline Máximo & 170 & 170 & 110 & 100 & 126 & 116 \\
\hline Valor de $p$ & \multicolumn{2}{|c|}{0,910} & \multicolumn{2}{|c|}{0,179} & \multicolumn{2}{|c|}{0,373} \\
\hline
\end{tabular}

Pressão Arterial Sistólica (PAS); Pressão Arterial Diastólica (PAD); Pressão Arterial Média (PAM).

A glicemia obtida na hora da consulta de Enfermagem não demonstrou ser um fator isolado para desenvolvimento de infecção. A média ultrapassou os $100 \mathrm{mg} / \mathrm{dL}$ nos dois grupos. O valor mínimo foi de $72 \mathrm{mg} / \mathrm{dL}$ para ambos os grupos. Já os valores máximos ficaram acima de $170 \mathrm{mg} / \mathrm{dL}$. $O$ valor de $p$ não demonstrou significância estatística.

A taxa de filtração glomerular obtida através da Equação de Cockcroft-Gault apresentou médias diferentes para GI e GNI, apesar do valor de p não ser estatisticamente significativo. Isto se deve ao fato de no GNI não ter pacientes com insuficiência renal, sendo que no GI havia dois pacientes com insuficiência renal diagnosticada, dos quais, um com dependência de hemodiálise. Estes pacientes tiveram taxa de filtração glomerular fora dos padrões, ocasionando grande desvio padrão neste grupo.

A comparação entre GI e GNI quanto à média de tempo de internamento do paciente no pré-operatório não demonstrou significância estatística. Os valores médios diferem mais de 10 horas entre os dois grupos, a mediana apresenta diferença de 47 horas, e o tempo mínimo de internamento pré-operatório variou em apenas uma hora entre 
os grupos. A diferença entre os valores máximos foi superior a 140 horas, sendo que o registro mais longo pertenceu ao $\mathrm{Gl}$ com um paciente internado por 552 horas no pré-operatório. 0 desvio padrão foi elevado nos dois grupos.

O tempo de cirurgia não demonstrou significância estatística quando comparado entre os dois grupos. Autilização de CEC variou entre os dois grupos. No GNI houve 4 pacientes que não necessitaram deste artifício durante a cirurgia. Já no Gl o uso de CEC foi uma constante com duração de uso de 38 a 131 minutos. Todavia, na análise estatística, não houve resultados com diferença significativa quando comparado os dois grupos. A Tabela 3 apresenta estas variáveis.

Tabela 3 - Características pré e transoperatórias

\begin{tabular}{|c|c|c|c|c|c|c|}
\hline Preditor & Grupo & Média & DP & Mínimo & Máximo & Valor $p$ \\
\hline \multirow{2}{*}{ Glicemia } & GI & 109,14 & 27,48 & 72 & 175 & 0,346 \\
\cline { 2 - 7 } & GNI & 102,42 & 26,28 & 72 & 172 & \\
\hline $\begin{array}{c}\text { Taxa de Filtração } \\
\text { Glomerular }\end{array}$ & GI & 75,15 & 20,52 & 19,3 & 98,1 & 0,227 \\
\cline { 2 - 7 } & GNI & 83,70 & 11,37 & 51,5 & 103 & \\
\hline \multirow{2}{*}{$\begin{array}{c}\text { Horas Int. Pré } \\
\text { Duração da Cirurgia } \\
\text { (min) }\end{array}$} & GI & 144,94 & 121,36 & 18 & 552 & 0,735 \\
\cline { 2 - 7 } & GNI & 131,62 & 105,72 & 19 & 408 & \\
\hline \multirow{2}{*}{\begin{tabular}{c} 
Tempo CEC \\
\cline { 2 - 7 }
\end{tabular}} & GNI & 309,76 & 84,59 & 210 & 540 & 0,689 \\
\cline { 2 - 7 } & GI & 77,95 & 37,24 & 0 & 132 & \\
\hline Circulação Extracorpórea (CEC). & & & & & & \\
\hline
\end{tabular}

A utilização de hemocomponentes quando comparados sem distinção, não demonstrou ser um fator predisponente para ISC. No entanto, ao comparar o GI e GNI na quantidade de concentrado de hemácias recebidas, houve significância estatística ( $p$ de 0,005$)$ demonstrando tendência para infecção no grupo $\mathrm{GI}$ onde a média de utilização foi de 3,90 unidades por paciente. A Tabela 4 representa estas variáveis.

Tabela 4 - Hemocomponentes trans e pós-operatório

\begin{tabular}{|c|c|c|c|c|c|c|c|c|}
\hline & \multicolumn{2}{|c|}{$\mathrm{CH}$} & \multicolumn{2}{|c|}{$\mathrm{CP}$} & \multicolumn{2}{|c|}{ PLASMA } & \multicolumn{2}{|c|}{ CRIO } \\
\hline & $\mathrm{Gl}$ & GNI & $\mathrm{Gl}$ & GNI & GI & GNI & $\mathrm{GI}$ & GNI \\
\hline Média & 3,90 & 2,14 & 2,80 & 4,66 & 1,57 & 1,90 & 0,38 & 0,42 \\
\hline Desvio Padrão & 2,62 & 1,52 & 8,98 & 8,73 & 2,42 & 2,77 & 1,74 & 1,36 \\
\hline Mínimo & 0 & 0 & 0 & 0 & 0 & 0 & 0 & 0 \\
\hline Máximo & 9 & 6 & 40 & 27 & 7 & 11 & 8 & 5 \\
\hline Valor de $p$ & & 05 & & 09 & & & & 25 \\
\hline
\end{tabular}

Concentrado de Hemácias (CH); Concentrado de Plaquetas (CP); Plasma Fresco (PLASMA); Crio Precipitado (CRIO).

Ao analisar o ODDS RATIO para os fatores que foram estudados, considerando o número de ocorrências em cada grupo para um determinado fator, foram evidenciadas variações relevantes. Os pacientes tabagistas tiveram 2,33 vezes maior chance de ter infecção, quando comparados com os pacientes não tabagistas ou ex-tabagistas que pararam de fumar há mais de 10 anos. Os pacientes que receberam crio precipitado, concentrado de plaquetas e plasma fresco tiveram menor índice de infecção quando comparados ao pacientes que não utilizaram estes hemocomponentes.

O uso de concentrado de hemácias, a circulação extracorpórea e o tempo de cirurgia mostraram tendência para infecção, sendo mais frequentes no $\mathrm{Gl}$, mas não foram suficientes para aumentar significativamente o risco relativo entre os grupos. Já o IMC elevado apresentou risco 1,33 vezes maior de ocorrer ISC e o tempo de internamento pré-operatório aumentou em 
1,43 vezes o risco da ISC. O Gráfico 1 apresenta estes valores.

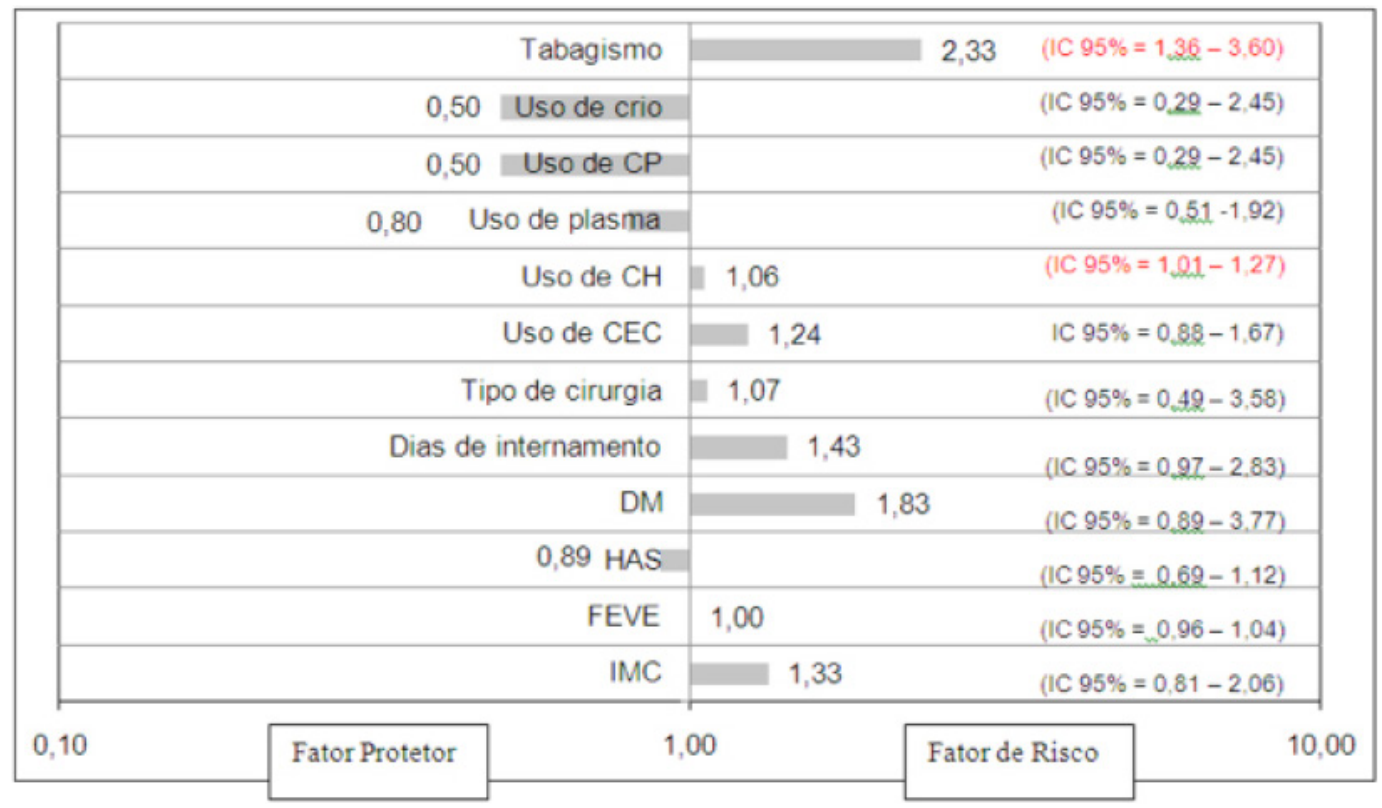

Gráfico 1 - ODDS RATIO dos fatores de risco levantados, IC 95\%.

Pacientes diabéticos tiveram quase duas vezes mais chance de infecção quando comparados com os pacientes não diabéticos. No tempo de observação não foi analisado o controle glicêmico, mas mesmo com glicemias similares, a presença da diabetes mostrou-se um fator predisponente para ISC.

A hipertensão arterial não se apresentou como um fator predisponente para infecção neste estudo, curiosamente apresentando uma tendência como fator protetor. A FEVE de baixos valores mostrou-se sem efeito quando avaliado entre GI e GNI, apresentando equivalência entre estes valores.

\section{Discussão}

As características encontradas nas populações do presente estudo foram semelhantes à de outros estudos consultados $^{2-4,6}$. As médias de idade para Gl e GNI, apesar do estudo aceitar pacientes acima dos 60 anos, ficou acima dos 70 anos, evidenciando o envelhecimento da população que se submete a cirurgias cardíacas. Destaca-se a importância da adequada abordagem ao paciente idoso, uma vez que o envelhecimento populacional torna a sociedade candidata à cirurgia cardíaca progressivamente menos resistente às infecções.

As características clínicas foram muito semelhantes entre os dois grupos, ou seja, uma amostra originalmente homogênea. Esta condição pode ter minimizado as diferenças entre as médias dos dados avaliados, que em sua grande maioria não apresentaram diferenças estatísticas, apesar da tendência para ISC em vários fatores estudados.

No GI e no GNI havia pacientes diabéticos, no entanto, não se observou descompensação glicêmica em nenhum dos grupos durante a consulta de Enfermagem. Isto demonstra a conscientização dos clínicos em manter os níveis glicêmicos estabilizados no pré-operatório. Mesmo assim, identificou-se um risco 1,83 vezes maior de infecção pela presença desta enfermidade. Tal achado está em concordância com os resultados apresentados nos estudos de Guaragna ${ }^{6}$ e Antoniali ${ }^{7}$, que embora retrospectivos, apresentam amostras maiores.

A taxa de filtração glomerular para diagnosticar insuficiência renal não apresentou relevância neste estudo. Sugere-se que tal fato se deve ao número reduzido de pacientes e ao fato de haver no $\mathrm{GI}$ dois pacientes com insuficiência renal diagnosticada, dos quais um com dependência de hemodiálise. Estes pacientes tiveram taxa de filtração glomerular fora dos padrões, ocasionando grande desvio padrão neste grupo e dificultando a análise.

Salienta-se que os riscos de complicações pós-operatórias já estão bem definidos em pacientes com insuficiência renal e a avaliação de um nefrologista deve ser considerada em todos os pacientes com creatinina sérica pré-operatória maior que $1,5 \mathrm{mg} / \mathrm{dL}^{8}$. 
Ambos os grupos apresentavam pacientes hipertensos, desta forma, houve casos de PAM acima de $115 \mathrm{mmHg}$. Devese considerar neste estudo que a consulta de Enfermagem foi realizada na noite anterior a cirurgia e que os valores pressóricos neste momento poderiam ser influenciados pelo estresse vivenciado pelos pacientes. Também não foi evidenciada relevância estatística quando comparado o grupo de hipertensos com o grupo de não hipertensos, tanto qualitativamente ou quantitativamente.

Os pacientes tabagistas receberam orientação para parar de fumar pelo menos uma semana antes da cirurgia. Como esta orientação não é apresentada como uma obrigatoriedade a todos os pacientes houve relatos de pacientes fumando no dia da cirurgia. Outros relatos demonstram que alguns pacientes tornaram a fumar assim que receberam alta da unidade de terapia intensiva. Desta forma, o grupo tabagista apresentou risco 2,33 vezes maior de apresentar ISC quando comparados aos pacientes não tabagistas. Este resultado corrobora com o estudo de Fatureto ${ }^{9}$ e encontra resultados semelhantes aos de Antoniali'.

A variável fração de ejeção obtida em todos os pacientes através de ecocardiograma transtorácico no pré-operatório não demonstrou ser um fator significante quando analisado separadamente.

Foram identificados pacientes com IMC acima de $30 \mathrm{~kg} / \mathrm{m}^{2}$, que são considerados obesos de acordo com a diretriz da Sociedade Brasileira de Endocrinologia Metabologia. Neste grupo houve uma tendência a ISC de 1,33 vezes maior. Em 2004, Guaragna et al., ${ }^{6}$ encontraram resultados semelhantes quando estudaram 1298 pacientes que haviam sido submetidos a cirurgia com esternotomia longitudianal.

Pessoas obesas (>20\% do peso ideal) apresentam maior incidência de infecção esternal. No estudo do Parisian Mediastinitis Study Group Risk Factors for Deep Sternal Wound Infective ${ }^{10}$, a obesidade foi o único fator de risco pré-operatório independente para infecção da cicatriz incisional.

O tempo de hospitalização pré-operatória variou entre 18 e 552 horas, com pacientes que ficaram mais de uma semana internados, aguardando a cirurgia proposta. 0 período de hospitalização pré-operatória é frequentemente associado ao aumento do risco do paciente desenvolver ISC, devido à colonização da pele pela microbiota hospitalar. Em pacientes com 7 dias de hospitalização prévia, os índices de ISC se tornam até duas vezes superiores aos com apenas 1 dia de internação ${ }^{3}$. Neste estudo, os pacientes que ficaram institucionalizados por mais de uma semana antes da cirurgia, tiveram 1,43 vezes mais risco de infecção quando comparados aos pacientes com menos de 7 dias internamento antes da cirurgia.

A revascularização do miocárdio é descrita como fator predisponente para infecção de sitio cirúrgico quando associada ao uso de mais de uma ponte de artéria torácica interna. A utilização de duas destas artérias é um fator muito discutido, pois predispõe à desvascularização do esterno, tornando-o um ambiente propício para o aparecimento de infecção, principalmente se estiver associado à obesidade e diabetes ${ }^{8}$.

Nos pacientes avaliados não houve significância estatística quando analisados os tempos de utilização da CEC, porém, quando comparados aos pacientes que não necessitaram deste instrumento durante 0 ato cirúrgico, notouse discreta tendência para ISC. A utilização de circulação extracorpórea é documentada como fator importante para infecção. Isto devido à síndrome de resposta inflamatória sistêmica, pois há liberação de mediadores inflamatórios pelo músculo cardíaco e pulmões. Essas alterações sistêmicas muitas vezes podem ser confundidas com quadro séptico ou pulmão de choque ${ }^{8}$.

$\mathrm{O}$ uso de hemocomponetes foi o dado que demonstrou certo desencontro com a literatura. $\mathrm{O}$ sangramento no trans e pós-operatório ocorrem por várias causas, entre elas a utilização da CEC, coagulopatias, uso de anti-agregante plaquetário recente e necessidade de re-operação. Independente do motivo de sangramento, na maioria das vezes tem-se necessidade de hemotransfusão. A utilização de concentrado de hemácias demonstrou significância estatística nos testes realizados $(p=0,005)$, demonstrando ser um fator favorecedor de ISC quando comparados $\mathrm{GI}$ e GNI, de acordo com os dados encontrados na literatura ${ }^{2,8,11}$.

No entanto, quando analisar o risco associado aos hemocomponentes responsáveis por fator de coagulação, notase tendência protetora para o uso de crio precipitado e concentrado de plaquetas. A comparação entre as quantidades destes hemocomponentes não teve significância estatística. Não se encontrou explicação plausível para estes dados, exceto pela casualidade de poucos pacientes do GI terem usado uma quantidade maior de unidades, enquanto o uso entre os pacientes do GNI foi mais regular, mas em menores quantidades.

A experiência deste estudo encontrou limitações na população estudada que foi acima de 60 anos, ainda encontrou restrições com o pareamento, devido o GI ser menor que o GNI. Para uma pesquisa ampla sugerimos estudo multicêntrico, assim podem ser estudas várias abordagens e populações de diversas idades, não só os fatores que a literatura classifica como preditor de infecção de Sítio cirúrgico. 


\section{Considerações finais}

A prevenção da infecção de sitio cirúrgico deve ser a principal atitude a ser tomada pela equipe de saúde no acompanhamento de pacientes que se submetem a procedimentos cirúrgicos.

Neste sentido, o estudo demonstrou que entre os fatores predisponentes para ISC avaliados, como IMC (abaixo do normal ou normal), FEVE, pressão arterial, índice de glicemia, taxa de filtração glomerular, tempo de duração da cirurgia, tempo de duração da CEC e quantidade de hemocomponentes não se apresentam com significância estatística, ou seja, não podem ser considerados potenciais preceptores da ISC.

No entanto, o tabagismo, internamento prolongado anterior a cirurgia, IMC elevado, e uso de concentrado de hemácias apresentaram-se com significância estatística, portanto, sendo considerados como fatores predisponentes a ISC.

Portanto, a existência de tais riscos deve ser alertada imediatamente a equipe multidisciplinar que acompanha 0 paciente, sobretudo, pela maior a probabilidade de infecção em sítio cirúrgico.

\section{Referências Bibliográficas}

1. Sasaki VDM, Romanzini AE, de Jesus APM, de Carvalho E, Gomes JJ, Damiano VB. Vigilância de infecção de sítio cirúrgico no pós-alta hospitalar de cirurgia cardíaca reconstrutora. Texto \& Contexto Enfermagem. 2011;20(2):328-32.

2. Gelape CL. Infecção do sítio operatório em cirurgia cardíaca. Arq Bras Cardiol. 2007;89(1):e3-9.

3. Silva QCG, Barbosa MH. Fatores de risco para infecção de sítio cirúrgico em cirurgia cardíaca. Acta paul enferm. 2012;25(spe2):89-95.

4. De Oliveira DC, Oliveira Filho JB, Silva RF, Moura SS, Silva DJ, Do Egito EST, et al. Sepse no pós-operatório de cirurgia cardíaca: descrição do problema. Arq Bras Cardiol. 2010;94(3):352-6.

5. Rosenthal VD, Maki DG, Jamulitrat S, Medeiros EA, Todi SK, Gomez DY, et al. International nosocomial infection control consortium (INICC) report, data summary for 2003-2008, issued June 2009. American journal of infection control. 2010;38(2):95-104. e2.

6. Guaragna JC, Facchi LM, Baião CG, Cruz I, Bodanese L, Albuquerque L, et al. Preditores de mediastinite em cirurgia cardíaca. Rev Bras Cir Cardiovasc. 2004;19(2):165-70.

7. Antoniali F, Costa CE, Tarelho LS, Lopes MM, Albuquerque APN, Reinert GAA, et al. O impacto de mudanças nas medidas de prevenção e no tratamento de infecções incisionais em cirurgia de revascularização do miocárdio. Rev Bras Cir Cardiovasc. 2005;20(4):382-91.

8. Abboud CS. Infecção em pós-operatório de cirurgia cardíaca. Rev Soc Cardiol Estado de Säo Paulo. 2001;11(5):91521.

9. Fatureto MC, Neves Junior M, Santana T. Mediastinite aguda. Análise retrospectiva de 21 casos. J Bel RD, Ivanov J, Cohen G, Scully HE, et al. Deep sternal wound infection: risk factors and outcomes. The Annals of thoracic surgery. 1998;65(4):1050-6.

11. Atik FA, Miana LA, Jatene FB, Auler Júnior JOC, Oliveira A. A cirurgia de revascularização do miocárdio sem circulação extracorpórea minimiza o sangramento pós-operatório e a necessidade transfusional. Arq Bras ras Pneumol. 2005;31(4):307-11.

10. Borger MA, Rao V, WeisCardiol. 2004;83(4):332-7.

\section{Elia Machado de Oliveira}

Endereço para correspondência - Rua José Ferreira Barros, 598. Vila Fanny, CEP 81030-320, Curitiba, PR, Brasil.

E-mail: elia561@hotmail.com

Currículo Lattes: http://lattes.cnpq.br/2209931172316428

Josue Bruginski de Paula - josuebrugipaula@gmail.com

Recebido em 15 de fevereiro de 2013.

Publicado em 30 de janeiro de 2014. 